\title{
Criminologie
}

\section{Les adolescentes membres des bandes marginales : un potentiel antisocial atténué par la dynamique de la bande?}

\section{Nadine Lanctôt et Marc LeBlanc}

Volume 30, numéro 1, printemps 1997

Criminalités économiques

URI : https://id.erudit.org/iderudit/017400ar

DOI : https://doi.org/10.7202/017400ar

Aller au sommaire du numéro

Éditeur(s)

Les Presses de l'Université de Montréal

ISSN

0316-0041 (imprimé)

1492-1367 (numérique)

Découvrir la revue

Citer cet article

Lanctôt, N. \& LeBlanc, M. (1997). Les adolescentes membres des bandes marginales : un potentiel antisocial atténué par la dynamique de la bande ? Criminologie, 30(1), 111-130. https://doi.org/10.7202/017400ar
Résumé de l'article

The goal of this article is to improve our knowledge concerning the social and personal characteristics of the female gang members. Data have been collected from 150 girls who were convicted by the juvenile court of Montreal during 1992 and 1993. The analysis shows that girls who join gangs have serious handicaps which are related to their social adaptation, their personality and their deviant and delinquent conducts. Consequently, female gang membership responds to a selection process, as it does with the male membership. The profile of the female also changes depending on the structure of the gang to which they join. As the gang becomes more organized, the girls' personality gets worst. However, the context of the organized gangs seems to limit the girls to auxiliary roles rather then being an opportunity to discharge their antisocial potential. 
LES ADOLESCENTES MEMBRES DES BANDES MARGINALES : UN POTENTIEL ANTISOCIAL ATTÉNUÉ PAR LA DYNAMIQUE DE LA BANDE ?

Nadine Lanctôt ${ }^{1}$

Marc LeBlanc ${ }^{2}$

The goal of this article is to improve our knowledge concerning the social and personal characteristics of the female gang members. Data have been collected from 150 girls who were convicted by the juvenile court of Montreal during 1992 and 1993. The analysis shows that girls who join gangs have serious handicaps which are related to their social adaptation, their personality and their deviant and delinquent conducts. Consequently, female gang membership responds to a selection process, as it does with the male membership. The profile of the female also changes depending on the structure of the gang to which they join. As the gang becomes more organized, the girls' personality gets worst. However, the context of the organized gangs seems to limit the girls to auxiliary roles rather then being an opportunity to discharge their antisocial potential.

L'influence des rôles sociaux est un thème qui retient particulièrement l'attention dans l'étude du phénomène des bandes chez les adolescentes. Il est d'abord soutenu que les rôles que la société attribue aux adolescentes limitent leur exposition aux bandes marginales (Thrasher, 1927 ; Crawford, Malamud et Dumpson, 1950 ; Cohen, 1955 ; Miller, 1958 ; Cloward et Ohlin, 1960 ; Brown, 1977 ; Campbell, 1984a ; Harris 1988). De plus, non seulement peu de filles sont-elles identifiées comme membres de bandes marginales, mais celles qui s'y joignent sont tenues de ne pas dépasser le seuil de la périphérie (Crawford et coll., 1950 ; Short, 1968 ; Miller, 1975 ; Collins, 1979 ; Sarnecki, 1986 ; Taylor, 1990 ; Joe et Chesney-Lind-Lind, 1995). Ce rôle d'auxiliaire limite généralement les comportements marginaux des filles à la consommation des psychotropes, à la promiscuité sexuelle, aux fugues, à la participation aux batailles entre bandes ainsi qu'au transport des armes (Crawford et coll., 1950 ; Ackley et Fliegel, 1960 ; Short, 1968 ; Miller, 1973 ;

1. M.Sc. (Criminologie), candidate au doctorat à l'École de criminologie et assistante de recherche au Groupe de recherche sur les adolescents en difficulté de l'École de psychoéducation, de Université de Montréal.

2. Ph.D. (Criminologie), professeur titulaire à l'École de psychoéducation et chercheur au Groupe de recherche sur les adolescents en difficulté de Université de Montréal. 
Haskins, 1974 ; Bowker et al., 1980, Campbell, 1984b ; Sarnecki, 1986 ; Fishman, 1988, Brotherton, 1996). Le contexte des bandes semble ainsi laisser peu de place aux initiatives délinquantes des adolescentes.

Par contre, avant d'être en mesure d'affirmer que la dynamique des bandes limite les initiatives délinquantes de ses membres féminins, il importe de s'assurer que ces adolescentes ont un réel potentiel de passage à l'acte. Or, très peu d'études s'intéressent aux difficultés d'adaptation des adolescentes qui participent à des bandes marginales. Il s'avère donc pertinent de vérifier si un processus de sélection soutient l'adhésion des filles aux bandes marginales. Chez les garçons, ce processus de sélection est accepté, puisque les adolescents qui se retrouvent membres de bandes sont ceux qui accusent les déficits les plus lourds (Lanctôt et Le Blanc, 1996b). Le profil des filles membres des bandes s'avère très peu documenté. Les quelques études qui traitent de l'adaptation sociale des filles rapportent que ces dernières rejettent les rôles féminins que la société leur confère (Thrasher, 1927 ; Crawford et coll., 1950 ; Campbell, 1984b ; Harris, 1988), qu'elles ont des activités et des intérêts limités (Crawford et coll., 1950), qu'elles ont peu de succès et d'aspirations scolaires (Campbell, 1984a ; Harris, 1988 ; Bjerregaard et Smith, 1993) et qu'elles investissent une grande partie de leur temps avec leurs copines (Bowker et Klein, 1983). Quant à la qualité des relations que ces adolescentes entretiennent avec leurs parents, elle demeure ambiguë. Bowker et Klein (1983) observent que ces relations ne sont pas affectées par l'appartenance à une bande, alors que Harris (1988) rapporte le contraire.

La personnalité des adolescentes qui sont membres d'une bande retient davantage l'attention des chercheurs. Crawford et coll. (1950) affirment d'abord que les filles quj participent à une bande se caractérisent par de profonds sentiments d'infériorité, ce qui les porte à se joindre à une bande dans le but d'être aidées et comprises. Brown (1977) soutient que l'adhésion à une bande trahit des besoins psychologiques de base, tel le désir d'être accepté. L'étude d'Arpin et coll. (1994) indique également que les adolescentes qui adhèrent à une bande marginale sont à la recherche de liens affectifs. L'utilisation des méthodes quantitatives ne retient toutefois pas la faible estime de soi comme un prédicteur significatif de l'adhésion à une bande (Bowker et Klein,1983 ; Bjerregaard et Smith,1993). L'inventaire de personnalité de Jesness, utilisé dans l'étude de Bowker et Klein (1983), dévoile enfin que les relations interpersonnelles posent de réelles difficultés aux filles membres de bandes. Malgré ces résultats concluants, Bowker et Klein ne reconnaissent toutefois pas le potentiel antisocial des filles membres des bandes. Ces auteurs affirment plutôt que ces résultats sont le fruit du racisme, du sexisme et de la pauvreté auxquelles se heurtent les adolescentes. Cette interprétation souligne l'importance que prennent les rôles sexuels dans 
l'analyse du phénomène des bandes chez les filles. Le potentiel de dysfonctionnalité de ces dernières se retrouve ainsi souvent effacé.

D'autre part, nous disposons de peu de connaissances sur la structure des bandes auxquelles participent les filles. Campbell (1984a) rapporte que les bandes féminines affiliées aux bandes des garçons sont peu structurées. La soljdarité de ces bandes s'effrite rapidement et leur organisation est instable. Mais qu'en est-il de l'influence de la structure de la bande sur l'adaptation sociale et personnelle des filles ainsi que sur leurs comportements déviants et délinquants ? Les filles qui adhèrent à des bandes structurées sontelles davantage dysfonctionnelles que celles qui participent à des bandes peu organisées ?

Enfin, la majorité des études font appel aux statistiques officielles ou aux agents du système de la justice pour repérer les membres des bandes (Thrasher, 1927 ; Cohen, 1955 ; Bowker et coll., 1980 ; Campbell, 1984b ; Sarnecki, 1986 ; Joe et Chesney-Lind, 1995). Or, sachant que les filles ont moins de contacts que les garçons avec la justice, il va de soi qu'elles soient moins nombreuses à être identifiées comme membres de bandes par les autorités officielles. D'autre part, puisque les adolescentes ne sont habituellement pas impliquées dans le noyau central de la bande, leur participation s'avère moins visible que celle des garçons (Sarnecki, 1986 ; Taylor, 1990). De surcroît, il serait erroné d'évaluer la participation des adolescentes aux bandes marginales selon le nombre de bandes féminines repérées. Les branches féminines affiliées aux bandes de garçons ne sont généralement pas suffisamment organisées pour être identifiées par les agences officielles (Lanctôt et Le Blanc, 1996a).

Le recours aux sondages est donc une solution à envisager. Les adolescentes rapportent ainsi elles-mêmes si elles sont impliquées ou non dans une bande marginale. Fagan (1990) affirme, à ce propos, que l'auto-révélation est une méthode efficace pour traiter du phénomène des bandes marginales parce que les adolescents sont sensibilisés au problème des bandes dans leur quartier. Les rares études qui utilisent cette méthode rapportent d'ailleurs des résultats complètement différents de ceux reposant sur les données des agences officielles quant à l'ampleur de la participation des filles aux bandes. Par exemple, l'enquête de Bjerregard et Smith (1993) auprès d'écoliers et d'écolières qui évoluent dans un quartier caractérisé par de hauts taux de délinquance rapporte que la proportion des filles qui participent aux bandes se rapproche grandement de celle des garçons ( $22 \%$ contre $20 \%$ ). Dans la même veine, un sondage effectué auprès des adolescentes d'un centre de réhabilitation indique que près de la moitié de ces filles se sont déjà reliées à des bandes marginales (Arpin et coll., 1994), une proportion semblable à celle obtenue pour les garçons judiciarisés (Lanctôt et Le Blanc, 1996b) 
L'utilisation des sondages semble donc démontrer que le phénomène des bandes marginales n'est pas aussi étranger aux filles que le laissent croire les données officielles. Cette proportion semblable de filles et de garçons qui participent aux bandes signifierait-elle que les filles qui adhèrent aux bandes sont aux prises, à l'instar des garçons, avec de réelles difficultés d'adaptation?

\section{SUJETS ET VARIABLES}

L'échantillon comprend 150 adolescentes qui ont reçu une ordonnance de la Chambre de la jeunesse de Montréal en vertu de la Loi sur la protection de la jeunesse (troubles de comportement sérieux : $83 \%$ ) ou de la Loi sur les jeunes contrevenants $(17 \%)$ entre les mois de février 1992 et de juin 1993. L'âge moyen de ces filles est de 15,1 ans. Les adolescentes en difficulté proviennent de divers groupes ethniques ; dans $22 \%$ des cas, au moins un des deux parents a immigré au Québec. Une entrevue d'une durée d'environ deux heures a été réalisée avec chacune des filles. Cette entrevue permet de dresser un profil social en fonction des dimensions importantes de la vie des adolescentes, soit la famille, l'école, les pairs, les activités et les valeurs morales. L'ampleur et la nature des troubles de comportements et des activités délictueuses sont aussi considérées. De plus, l'adaptation personnelle est mesurée à l'aide des inventaires de personnalité de Jesness et d'Eysenck. La qualité métrique de ces échelles a été largement démontrée (Le Blanc, McDuff, Fréchette,1994).

Une question de l'entrevue porte spécifiquement sur la participation des adolescentes aux bandes marginales. Au total, $69 \%(\mathrm{n}=103)$ des adolescentes en difficulté répondent par l'affirmative à la question suivante : Astu đéjà fait partie d'un groupe de jeunes (gang) qui fait des mauvais coups ? D'une façon plus précise, $31 \%(\mathrm{n}=47)$ des filles de l'échantillon n'ont jamais fait partie d'une bande marginale, $19 \%(n=29)$ se sont déjà affiliées à une bande sans toutefois que leur participation persiste durant la dernière année, $17 \%(n=25)$ viennent de se joindre récemment à une bande et le tiers des filles $(n=49)$ participent à une bande depujs plus de deux ans. Ces adolescentes n'adhèrent pas toutes à des bandes dont l'organisation est définie. Le degré de structure des bandes auxquelles particjpent les adolescentes est établi en fonction du nombre de caractéristiques que rapporte chacune d'elles quant à leur bande. Les membres des bandes rapportent ainsi si leur bande a un chef, un lieu de rencontre précis, des signes distinctifs, un nom ou une initiation. Parmi celles qui ont adhéré à une bande au cours de la dernière année, $28 \%(\mathrm{n}=21)$ se lient à des bandes peu structurées $(0$ ou 1 des descripteurs ci-dessus), $42 \%(\mathrm{n}=31)$ participent à des bandes dont la 
structure est moyenne ( 2 ou 3 des caractéristiques) et $31 \%(n=22)$ se retrouvent dans des bandes structurées ( 4 ou 5 des traits).

Nous avons comparé d'abord les adolescentes qui ont déjà participé à une bande marginale et celles qui disent ne s'être jamais liées à une telle bande. Afin de vérifier si le processus de sélection observé chez les garçons se retrouve chez les filles. Ce processus implique que la bande recrute les individus dont la dysfonctionnalité est la plus marquée, et ce, tant au plan personnel et social que comportemental (Lanctôt et Le Blanc, 1996b). Dans le but d'évaluer si l'adhésion à une bande structurée trahit davantage de dysfonctionnalité que la participation à une bande moins organisée, nous avons comparé ensuite les adolescentes en fonction de la structure des bandes auxquelles elles participent. Afin de répondre à ces deux questions, des tests de différences des moyennes sont effectués, de même que l'analyse discriminante.

\section{QUI SONT LES ADOLESCENTES MEMBRES DES BANDES ?}

Le tableau 1 présente les résultats des adolescentes membres des bandes $(n=103)$ et de ceux des non-membres $(n=47)$ sous une forme standardisée en fonction de l'âge des adolescentes. Le corridor normatif se situe entre 40 et 60 . Des résultats hors de ce corridor trahissent la présence de réelles difficultés d'interaction avec le milieu. L'adaptation sociale des adolescentes est d'abord traitée, puis leur adaptation personnelle et leurs activités marginales.

\section{ADAPTATION SOCIALE}

L'appartenance ou non à une bande affecte très peu le profil familial des adolescentes de la Chambre de la jeunesse. En effet, seule la supervision parentale distingue la famille des deux groupes d'adolescentes. Les parents des membres des bandes surveillent peu les allées et les venues de leurs filles $(t=2,20 ; p<0,05)$. Les données du tableau 1 démontrent donc davantage de ressemblances que de différences quant à la famille des adolescentes des deux groupes. Des ressemblances ressortent également au niveau des liens qui unissent les adolescentes en difficulté à leur entourage. Les rapports entretenus avec la famille et avec les pairs se comparent en effet d'un groupe à l'autre, les premiers faisant défaut et les seconds étant plus serrés. Qu'elles soient membres ou non d'une bande marginale, les adolescentes en difficulté sont peu attachées à leur famille et elles investissent peu de temps dans les activités familiales. Ces adolescentes préfèrent se trouver en présence de leurs pairs qu'en présence de leur famille. Le temps passé avec les pairs incite par contre davantage les membres des bandes que les non-membres aux activités marginales. Bien que les activités que pratiquent les adolescentes 


\section{Tableau I}

Adolescentes membres et non-membres des bandes marginales

\begin{tabular}{|c|c|c|c|c|c|c|}
\hline \multirow[b]{2}{*}{ Variables } & \multirow[b]{2}{*}{ t ou X2 } & \multirow[b]{2}{*}{$\mathbf{p}$} & \multicolumn{2}{|c|}{$\begin{array}{c}\text { non-membres } \\
n=47\end{array}$} & \multicolumn{2}{|c|}{$\begin{array}{c}\text { membres } \\
\mathrm{n}=103\end{array}$} \\
\hline & & & moy. & é.-t. & moy. & é.-t. \\
\hline \multicolumn{7}{|l|}{ Statut } \\
\hline âge & 1,93 & 0.056 & 15,45 & $(1,52)$ & 15,01 & $(1,27)$ \\
\hline dépendance économique & $-0,24$ & 0,804 & 59,49 & $(11,71)$ & 60,02 & $(12,59)$ \\
\hline scolarité des parents & 0,50 & 0,620 & 53,55 & $(9,04)$ & 52,64 & $(9,33)$ \\
\hline mère travail & $-0,63$ & 0.532 & 57,09 & $(14,51)$ & 58,67 & $(13,65)$ \\
\hline immigration des parents & 0,70 & 0,704 & $26,7 \%$ & & $20,8 \%$ & \\
\hline séparation & 0.15 & 0,694 & $85,1 \%$ & & $82,5 \%$ & \\
\hline durée séparation & 0,36 & 0,720 & 85,03 & $(21,20)$ & 83,70 & $(20,75)$ \\
\hline fratrie & 1,22 & 0,223 & 42,66 & $(5,30)$ & 41,50 & $(5,23)$ \\
\hline suivi par travailleur social & 1,39 & 0,238 & $97,8 \%$ & & $93,2 \%$ & \\
\hline comparution tribunal & 0,18 & 0,671 & $95,7 \%$ & & $97,1 \%$ & \\
\hline désavantage familial & $-0,02$ & 0,984 & 59,98 & $(8,50)$ & 60,01 & $(7,75)$ \\
\hline désavantage socio-économique & 0,20 & 0,840 & 63.00 & $(13,43)$ & 62,50 & $(13.74)$ \\
\hline \multicolumn{7}{|l|}{ Famille } \\
\hline conflits conjugaux & 0.12 & 0,907 & 59,04 & $(15,87)$ & 58,58 & $(14,89)$ \\
\hline attachement & 0,50 & 0,619 & 43,14 & $(13,28)$ & 42,06 & $(11,63)$ \\
\hline investissement & 1,46 & 0.146 & 46,33 & $(9,54)$ & 43,44 & $(11,79)$ \\
\hline supervision & 2,20 & 0,029 & 44,08 & $(14,83)$ & 38,44 & $(14,26)$ \\
\hline règlements & $-0,08$ & 0,940 & 49,04 & $(8,52)$ & 49.16 & $(10.04)$ \\
\hline punitions & $-0,87$ & 0,387 & 57,43 & $(12,02)$ & 59,59 & $(14,86)$ \\
\hline déviance parentale & $-1,53$ & 0,127 & 48,22 & $(12,70)$ & 51,39 & $(11,11)$ \\
\hline normes parentales déviantes & 0,69 & 0.491 & 49,12 & $(11,37)$ & 47,88 & $(9,20)$ \\
\hline \multicolumn{7}{|l|}{ École } \\
\hline performance & $-0,19$ & 0,847 & 51,32 & $(9,68)$ & 51,73 & $(11,80)$ \\
\hline stress & $-2,19$ & 0,031 & 46,15 & $(10,46)$ & 50,85 & $(11,96)$ \\
\hline attachement & $-0,47$ & 0,642 & 43,85 & $(9,01)$ & 44.75 & $(10.89)$ \\
\hline investissement & 2,58 & 0,011 & 49,17 & $(17,16)$ & 42,55 & $(11,79)$ \\
\hline engagement & 0.19 & 0,851 & 47,85 & $(12,79)$ & 47,40 & $(12,94)$ \\
\hline sanctions & $-3,20$ & 0,002 & 53,01 & $(13,67)$ & 60,70 & $(13,47)$ \\
\hline décrochage & 0,12 & 0,729 & $10.6 \%$ & & $12.6 \%$ & \\
\hline \multicolumn{7}{|l|}{ Pairs } \\
\hline réseau & 1,13 & 0,260 & 53,66 & $(12,45)$ & 51,23 & $(11,99)$ \\
\hline investissement & $-0,86$ & 0,391 & 59,12 & $(8,09)$ & 60.33 & $(7,83)$ \\
\hline ami & $-0,93$ & 0,357 & 54,52 & $(6,94)$ & 56.30 & $(8,81)$ \\
\hline exposition aux pairs marginaux & $-8,22$ & 0,000 & 52,70 & $(8,65)$ & 68,07 & $(11,24)$ \\
\hline \multicolumn{7}{|l|}{ Activités } \\
\hline argent de poche & -1.27 & 0,208 & 46,52 & $(9,91)$ & 48,58 & $(8,78)$ \\
\hline activités sociales & $-1,66$ & 0.100 & 56,26 & $(9,59)$ & 59,14 & $(8,91)$ \\
\hline activités passives & 0,91 & 0,365 & 53,60 & $(10,06)$ & 52.05 & $(9.32)$ \\
\hline activités de participation & 1,80 & 0,074 & 52,44 & $(10,06)$ & 52,05 & $(9.32)$ \\
\hline travail & 1,17 & 0,242 & 52,94 & $(11,12)$ & 49,26 & $(9,29)$ \\
\hline flânerie & $-2,11$ & 0.036 & 46,84 & $(12,99)$ & 51,93 & $(13,79)$ \\
\hline fréquentation d'arcades & -5.04 & 0.000 & 51,45 & $(12,00)$ & 68,13 & $(18.80)$ \\
\hline
\end{tabular}


Tableau 1 (suite)

\begin{tabular}{|c|c|c|c|c|c|c|}
\hline \multirow[b]{2}{*}{ Variables } & \multirow[b]{2}{*}{$\operatorname{ton} \times 2$} & \multirow[b]{2}{*}{$\mathbf{p}$} & \multicolumn{2}{|c|}{$\begin{array}{c}\text { non-membres } \\
n=47\end{array}$} & \multicolumn{2}{|c|}{$\begin{array}{l}\text { membres } \\
n=103\end{array}$} \\
\hline & & & moy. & é.-t. & moy. & é.-t. \\
\hline \multicolumn{7}{|l|}{ Normes } \\
\hline adhésion aux normes & 2,80 & 0,006 & 48,83 & $(11,51)$ & 42.40 & $(13,51)$ \\
\hline respect de l'autorité & 3,03 & 0,003 & 45,09 & $(12,37)$ & 38,71 & $(11,63)$ \\
\hline neutralisation & $-2,16$ & 0,032 & 45,89 & $(11,41)$ & 50,07 & $(10,64)$ \\
\hline \multicolumn{7}{|l|}{ Adaptation personnelle } \\
\hline mésadaptation sociale & $-2,71$ & 0,008 & 55,89 & (14.94) & 62,75 & $(12,47)$ \\
\hline orientation aux valeurs & $-1,81$ & 0.072 & 57,14 & $(14,64)$ & 61.42 & $(11,26)$ \\
\hline autisme & $-1,36$ & 0,176 & 58,03 & $(14,45)$ & 61,46 & $(12,65)$ \\
\hline aliénation & $-2,14$ & 0,034 & 56,58 & $(15.22)$ & 62,31 & $(13,49)$ \\
\hline agressivité manifeste & $-3,05$ & 0,003 & 54,17 & $(12,84)$ & 60,87 & $(10,89)$ \\
\hline retrait & $-0,12$ & 0,904 & 58.04 & $(11,89)$ & 58,29 & $(10,37)$ \\
\hline anxiété sociale & $-0,18$ & 0,856 & 48,68 & $(12,95)$ & 49,07 & $(10,66)$ \\
\hline repliement & 1,89 & 0.061 & 47,41 & $(9,77)$ & 44,23 & $(8,41)$ \\
\hline déni & $-0,04$ & 0,967 & 41,63 & $(11,48)$ & 41,71 & $(9,18)$ \\
\hline psychotisme & $-1,41$ & 0,160 & 75,88 & $(13,48)$ & 79,22 & $(11,90)$ \\
\hline extraversion & $-2,78$ & 0,006 & 56,11 & $(10,03)$ & 60,97 & $(8,81)$ \\
\hline névrotisme & $-0,47$ & 0,637 & 51,63 & $(11,96)$ & 52,58 & $(9,82)$ \\
\hline \multicolumn{7}{|l|}{ Troubles de comportement } \\
\hline troubles de comportement & $-3,52$ & 0,001 & 60,59 & $(11.14)$ & 67,75 & $(11,61)$ \\
\hline rébellion familiale & $-1,30$ & 0,197 & 58,40 & $(13,03)$ & 61,49 & $(13,57)$ \\
\hline inadaptation scolaire & $-3,52$ & 0.001 & 49,39 & $(9,47)$ & 55,76 & $(10,50)$ \\
\hline promiscuité sexuelle & $-0,94$ & 0,348 & 59,10 & $(6,22)$ & 60.23 & $(7,01)$ \\
\hline drogues et alcool & $-3,44$ & 0,001 & 58.52 & $(12,36)$ & 66.91 & $(14,33)$ \\
\hline \multicolumn{7}{|l|}{ Délinquance } \\
\hline délinquance criminelle & $-5,36$ & 0,000 & 52,15 & $(12,83)$ & 67,43 & $(17,30)$ \\
\hline agression & $-5,45$ & 0,000 & 53,34 & $(13,35)$ & 69,52 & $(18,03)$ \\
\hline vandalisme & $-3,54$ & 0,001 & 51,64 & $(13,07)$ & 63,24 & $(20,41)$ \\
\hline vol mineur & $-2,30$ & 0,023 & 50,28 & $(11,64)$ & 55,57 & $(13,47)$ \\
\hline vol grave & $-2,81$ & 0,006 & 54,14 & $(15,56)$ & 64,99 & $(23,44)$ \\
\hline délinquance grave & $-4,29$ & 0,000 & 54,91 & $(15.57)$ & 71,13 & $(22,84)$ \\
\hline
\end{tabular}

des deux groupes se ressemblent plus qu'elles ne se distinguent, les filles membres des bandes sont davantage exposées, par le biais de leurs pairs (exposition aux pairs marginaux : $t=-8,22 ; p=0,000$ ) et de leurs activités (arcades : $t=-5,04 ; p=0,000$ ), à des influences déviantes. Cette exposition aux figures et aux lieux marginaux est facilitée par la supervision parentale relâchée à laquelle les membres des bandes ont surtout droit.

D'autre part, les filles membres des bandes sont peu réceptives aux contraintes sociales. Le résultat de ces adolescentes à l'adhésion aux normes est problématique $(t=2,80 ; p<0,01)$. Ainsi, les filles qui se joignent aux bandes rejettent les normes qui leur sont proposées en plus de ne pas s'imposer de barrières morales afin d'éviter la transgression des règles. La faible 
réceptivité aux contraintes s'explique également par le peu de respect qu'accordent les membres des bandes aux personnes en autorité $(t=3,03 ; p<$ $0,01)$. Les nombreuses sanctions reçues à l'école en témoignent d'ailleurs ( $t$ $=-3,20 ; p<0,01)$. Ces réprimandes ne sont pas le fruit de mauvaises notes, puisque les performances scolaires des membres des bandes sont dans la moyenne, tout comme celles des non-membres. Les adolescentes qui participent aux bandes marginales sont cependant moins impliquées à l'école que celles qui ne se joignent pas aux bandes. L'investissement scolaire faible $(t=2,58 ; p<0,05)$ témoigne du peu de temps que les membres des bandes consacrent aux activités scolaires et parascolaires.

Bref, les adolescentes en difficulté, et particulièrement celles qui s'affilient à des bandes marginales, ne s'abritent pas autant derrière leurs rôles sociaux que le prétend la littérature. Bien que de nombreuses ressemblances s'observent entre les adolescentes judiciarisées qui adhèrent aux bandes et celles qui ne s'y sont jamais liées, les barrières les plus étanches contre l'activité marginale ne sont pas suffisamment solides chez celles qui participent aux bandes pour que leur conformité sociale soit assurée. La faible réceptivité aux contraintes sociales, la supervision parentale inadéquate ainsi que l'exposition aux influences marginales trahissent des difficultés d'adaptation sociale accrues chez les adolescentes qui adhèrent aux bandes. Ces mêmes variables caractérisent les garçons membres de bandes (Lanctôt et Le Blanc, 1996b). Les filles qui se lient aux bandes marginales éprouvent donc des difficultés d'adaptation similaires à celles de leurs confrères.

\section{ADAPTATION PERSONNELLE}

L'analyse de la personnalité, à partir des inventaires de personnalité de Jesness et d'Eysenck, révèle que les adolescentes membres des bandes sont celles qui accusent le plus de déficits. En effet, ces dernières affichent les résultats les plus dysfonctionnels pour les quatre traits de personnalité qui distinguent significativement les adolescentes des deux groupes. Ces dimensions sont la mésadaptation sociale $(t=-2,71 ; p<0,01)$, l'aliénation $(t=$ $-2,14 ; p<0,05)$, l'agressivité manifeste $(t=-3,05 ; p<0,01)$ et l'extraversion $(t=-2,78 ; p<0,01)$.

Le résultat élevé des membres des bandes au chapitre de la mésadaptation sociale indique que ces adolescentes ont de la difficulté à atteindre les exigences de façon socialement acceptable et qu'elles font preuve de peu de savoir-faire social. Elles atteignent leurs fins par l'opposition, par la provocation et par la destruction. La fibre morale des membres des bandes est donc lourdement affaiblie. Cette conscience sociale inadéquate est un révélateur majeur du potentiel de passage à l'acte. Les résultats des filles membres des 
bandes à l'aliénation et à l'agressivité manifeste renforcent leur potentiel de passage à l'acte. Le premier indice témoigne d'une intolérance soupçonneuse envers autrui alors que le second trahit une amertume vindicative. Les membres des bandes ont donc des réactions de méfiance envers autrui et elles se sentent envahies par des sentiments de colère. Le potentiel d'agressivité et d'emportement est donc au cour de la personnalité des adolescentes qui se lient à des bandes marginales. Bowker et Klein (1983) en sont venus à des résultats semblables à partir de l'inventaire de personnalité de Jesness. Ces derniers rapportent que les adolescentes qui font partie d'une bande ont de la difficulté à satisfaire aux exigences d'une façon socialement approuvée, qu'elles sont méfiantes vis-à-vis les figures d'autorité et qu'elles sont impulsives. Enfin, l'inventaire d'Eysenck démontre que les membres des bandes se démarquent par leur extraversion. Ces adolescentes recherchent ainsi les sensations fortes dans une dynamique de domination et d'insouciance.

La capacité de fonctionnalité sociale des adolescentes qui participent aux bandes marginales est donc lacunaire. Ces filles s'opposent aux normes sociales, elles se positionnent contre autrui et leur ressentiment est prêt à se déverser à tout moment. Le profil personnel des adolescentes membres de bandes va donc au-delà d'une faible estime de soi. Les études qui abordent la personnalité des adolescentes membres de bandes mettent surtout l'accent sur cette dimension et elles négligent d'évaluer le potentiel d'agressivité de ces dernières (Crawford et coll., 1950 ; Brown, 1977 ; Cambpell, 1990). Les résultats du tableau 1 démontrent pourtant que le dysfonctionnement personnel des adolescentes qui se lient aux bandes est bien réel, tout comme celui des garçons membres des bandes. En effet, les modes d'interaction privilégiés par ces derniers reposent principalement sur l'antagonisme, sur la méfiance et sur le désir de domination (Lanctôt et Le Blanc, 1996b).

\section{ACTIVITÉS MARGINALES}

Les activités marginales réfèrent à deux catégories de conduites : les troubles de comportement et les agirs délinquants. La déviance réfêre aux conduites qui violent les standards socialement approuvés, tandis que les conduites délinquantes se rapportent aux comportements qui violent la loi. Pour chacune des conduites présentées au tableau 1, la participation actuelle est rapportée. Ces résultats portent sur les comportements pratiqués au cours des douze derniers mois par les adolescentes en difficulté.

L'indice général des troubles de comportement indique que les filles qui participent aux bandes ont manifesté différentes formes d'activités déviantes au cours de la dernière année $(t=-3,52 ; p<0,01)$. Cette variété s'observe plus précisément au niveau de l'inadaptation scolaire $(t=-3,52$; 
$p<0,01)$ et au niveau de la consommation de drogues et d'alcool $(t=$ $-3,44 ; p<0,01)$. Les membres des bandes s'opposent ainsi de diverses façons à l'école et elles consomment une plus grande variété de drogues que leurs consceurs qui ne s'affilient pas aux bandes. L'engagement dans la délinquance est également marqué chez les filles qui participent aux bandes. Le bilan de la dernière année est lourd pour ces filles membres des bandes, puisqu'elles ont fait à l'expérience d'une grande variété d'actes délinquants $(t=-5,36$; $p=0,000$ ). Cette variété s'observe pour tous les types de délits. Les comportements des filles qui participent aux bandes sont donc pluse variés que ne le suggère la littérature (Crawford et coll., 1950 ; Ackley et Fliegel, 1960 ; Short, 1968 ; Miller, 1973 ; Sarnecki, 1986). Ces comportements ne se restreignent pas à la participation aux batailles et au transport des armes. Les filles membres des bandes ont à leur actif un grand répertoire d'actes déviants et délinquants.

En somme, les filles qui participent aux bandes ont un profil beaucoup plus sombre que celui des adolescentes qui ne font pas partie de bandes marginales. Les filles membres des bandes sont fortement exposées à des modèles déviants et elles pratiquent des activités qui ne favorisent pas la conformité sociale. Le manque d'encadrement parental de ces adolescentes de même que le peu de temps qu'elles accordent à l'école nuisent également à leur conformité sociale. Du côté de la personnalité, les membres des bandes se caractérisent par une conscience morale déficiente et par un fort potentiel d'agressivité et d'emportement. Ces conditions sociales et personnelles rendent donc les membres des bandes plus sensibles aux influences déviantes. Les comportements déviants et délinquants sont divers et marqués.

En effet, les filles qui participent aux bandes mettent à exécution des comportements marginaux de tous genres, allant des actes les plus bénins aux plus graves. Cette conclusion diffère de celle qui émane de la littérature. De nombreux auteurs rapportent en effet que le rôle secondaire des filles limite leurs comportements à la consommation de drogues et d'alcool, à la promiscuité sexuelle, aux fugues, à la participation aux batailles ainsi qu'au transport des armes (Crawford et coll., 1950 ; Ackley et Fliegel, 1960 ; Short, 1968 ; Miller, 1973 ; Haskins, 1974 ; Bowker et coll., 1980, Campbell, 1984b ; Sarnecki, 1986 ; Fishman, 1988). La divergence des conclusions s'explique peut-être par l'incapacité de notre analyse à distinguer les comportements commis dans le contexte de la bande de ceux qui se manifestent indépendamment de ce contexte, ce que ne font pas toujours les études citées. Une fille peut participer à un délit avec sa bande comme elle peut commettre un délit sans le soutien de celle-ci.

Malgré cette limite, il est possible d'affirmer que le phénomène des bandes chez les filles répond à un processus de sélection, au même titre que 
chez les garçons (Lanctôt et Le Blanc, 1996b). Les adolescentes en difficulté qui participent aux bandes sont celles qui sont aux prises avec les difficultés d'adaptation les plus marquées. L'analyse discriminante confirme ces observations. Cette analyse inclut les variables qui distinguent les membres et les non-membres des bandes avec un degré de probabilité inférieur ou égal à 0,10 , tout en n'impliquant pas de redondance. Huit prédicteurs permettent d'expliquer $30 \%$ de la variance, le Wilks' lambda est de 0,70 . Il est intéressant de noter le modèle construit pour les garçons affiche un pouvoir de discrimination semblable, puisque $32 \%$ de la variance y est expliquée (Lanctôt et Le Blanc, 1996b). L'agression physique est le prédicteur le plus puissant pour les filles comme pour les garçons. Ces rapprochements entre le profil des membres féminins et celui des membres masculins signifient-ils que les bandes exercent une influence similaire chez les filles et les garçons?

\section{L'INFLUENCE DE LA STRUCTURE DES BANDES}

Peu de connaissances sont disponibles sur la structure des bandes auxquelles participent les filles. Campbell (1984a) rapporte que les bandes féminines affiliées aux bandes de garçons sont peu structurées. La solidarité de ces bandes s'effrite rapidement et leur organisation est instable. Mais estce que l'adaptation sociale et personnelle et les comportements marginaux des filles varient en fonction de la structure des bandes ? Les filles qui adhèrent à des bandes structurées sont-elles davantage dysfonctionnelles que celles qui participent à des bandes peu organisées? Afin de vérifier si le dysfonctionnement des filles est plus élevé là où la structure des bandes est mieux définie, les profils des adolescentes sont comparés selon le niveau de structure des bandes auxquelles elles se joignent. Les résultats sont présentés au tableau 2 .

\section{ADAPTATION SOCIALE}

Seules trois variables familiales distinguent significativement les groupes d'adolescentes. D'abord, les parents des filles qui adhèrent aux bandes les plus structurées sont davantage dépendants au plan économique $(F=5,32$; $d l=2 ; p<0,01)$ et ils sont peu instruits $(F=5,66 ; d l=2 ; p<0,01)$. Ensuite, bien que les résultats ne dépassent pas le seuil de la normalité, la déviance caractérise davantage les parents des filles qui adhèrent aux quasi-bandes ou aux bandes structurées $(F=6,22 ; d l=2 ; p<0,01)$. Pour le reste, la situation familiale des filles des trois groupes est sensiblement la même, peu importe la structure des bandes auxquelles elles adhèrent. La qualité des relations familiales fait défaut chez les membres de chaque niveau de structure de même que la supervision parentale. Les filles de chaque groupe vivent 
Tableau 2

Comparaison des adolescentes selon la structure de leur bande

\begin{tabular}{|c|c|c|c|c|c|c|c|c|}
\hline \multirow[b]{2}{*}{ Variables } & \multirow[b]{2}{*}{ F/X2 } & \multirow[b]{2}{*}{$\mathbf{p}$} & \multicolumn{2}{|c|}{$\begin{array}{c}\text { réseaux } \\
\mathbf{n}=\mathbf{2 1}\end{array}$} & \multicolumn{2}{|c|}{$\begin{array}{c}\text { quasi-bandes } \\
\mathbf{n}=\mathbf{3 1} \\
\end{array}$} & \multicolumn{2}{|c|}{$\begin{array}{c}\text { bandes struct. } \\
\qquad \mathrm{n}=\mathbf{2 2}\end{array}$} \\
\hline & & & moy. & é.t.t. & moy. & é.-t. & moy. & é.t. \\
\hline \multicolumn{9}{|l|}{ Statut } \\
\hline âge & 2,14 & 0.1249 & 14,76 & $(1.09)$ & 15,06 & $(1,29)$ & 14.36 & $(1.22)$ \\
\hline dépendance économique & 5.32 & 0,0071 & 54.03 & $(10,46)$ & 60.24 & $(12,82)$ & 66.50 & $(13,00)$ \\
\hline scolarité des parents & 5,66 & 0,0056 & 54.92 & $(9,50)$ & 56,31 & (8.93) & 47,82 & $(8,05)$ \\
\hline mère travail & 0.68 & 0,5102 & 55.09 & $(11,60)$ & 59.43 & $(13.68)$ & 58.70 & $(13,53)$ \\
\hline immigration des parents & 3,95 & 0.4123 & $19.1 \%$ & & $27,6 \%$ & & $13.6 \%$ & \\
\hline séparation & 0.73 & 0,6936 & $76.2 \%$ & & $80.6 \%$ & & $86.4 \%$ & \\
\hline durée séparation & 1,14 & 0.3255 & 78.86 & $(22.37)$ & 82.13 & $(23,47)$ & 88,66 & $(18,06)$ \\
\hline fratrie & 0,96 & 0.3872 & 42.63 & $(5,63)$ & 40,88 & $(3,64)$ & 41.61 & $(4,23)$ \\
\hline suivi par travailleur social & 0,33 & 0,8490 & $95.2 \%$ & & $93.5 \%$ & $90,9 \%$ & & \\
\hline comparution tribunal & 1.41 & 0.4950 & $100,0 \%$ & & $96.8 \%$ & & $100,0 \%$ & \\
\hline $\begin{array}{l}\text { désavantage familial } \\
\text { désavantage socio- }\end{array}$ & 1,53 & 0.2239 & 57.65 & $(10.37)$ & 61.40 & $(8.10)$ & 58.34 & $(6.21)$ \\
\hline économique & 2.82 & 0.0664 & 57,33 & $(10.62)$ & 61.77 & $(14.97)$ & 67.05 & $(12,30)$ \\
\hline \multicolumn{9}{|l|}{ Familie } \\
\hline conflit conjugaux & 3.11 & 0,0553 & 53,14 & $(13,14)$ & 56.35 & $(12,70)$ & 66.51 & $(15,96)$ \\
\hline attachement & 0.37 & 0.6928 & 41.88 & $(12,21)$ & 39,69 & $(10.74)$ & 39.21 & $(9.96)$ \\
\hline investissement & 0,22 & 0,8055 & $42.2 !$ & $(7.51)$ & 42,08 & $(12.31)$ & 40,37 & $(10.14)$ \\
\hline supervision & 0.40 & 0,6750 & 38.65 & $(14,82)$ & 35,18 & $(13.72)$ & 37,20 & (13.84) \\
\hline règlements & 1,27 & 0,2872 & 49.16 & $(11,90)$ & 49.13 & $(10,39)$ & 53,44 & $(9.59)$ \\
\hline punitions & 1.99 & 0,1438 & 57.79 & $(16,06)$ & 60.91 & $(14.34)$ & 66.62 & $(14.18)$ \\
\hline déviance parentale & 6,22 & 0,0032 & 45,38 & $(6,29)$ & 53,71 & $(13,06)$ & 56,90 & (II.62) \\
\hline normes parentales déviantes & 0.16 & 0.8508 & 48.47 & $(6,89)$ & 48.42 & $(11,15)$ & 49.88 & $(10,62)$ \\
\hline \multicolumn{9}{|l|}{ Ecole } \\
\hline performance & 0.82 & 0.4445 & 48.14 & $(10.28)$ & 51,12 & $(10.55)$ & 52.61 & $(12.16)$ \\
\hline stress & 0,52 & 0.5949 & 49,82 & $(11,71)$ & 53,22 & $(10,94)$ & 53,35 & $(10.29)$ \\
\hline attachement & 0.47 & 0.6300 & 41,82 & $(7,93)$ & 44,87 & $(12,47)$ & 44.24 & $(10.07)$ \\
\hline investissement & 3.06 & 0,0536 & 39.99 & $(12,04)$ & 39.35 & $(9.67)$ & 46.98 & $(12,73)$ \\
\hline engagement & 2.31 & 0.1073 & 50.46 & $(12.07)$ & 43,48 & $(11.29)$ & 43,05 & $(13,16)$ \\
\hline sanctions & 1.57 & 0,2146 & 59,91 & (12.98) & 63.48 & $(12.64)$ & 66.89 & $(13.21)$ \\
\hline décrochage & 1,19 & 0.5507 & $14.3 \%$ & & $9.7 \%$ & & $4.5 \%$ & \\
\hline
\end{tabular}

Pairs

Comparaison des adolescentes

selon la structure de leur bande

\begin{tabular}{|c|c|c|c|c|c|c|c|c|}
\hline réseau & 0,35 & 0.7051 & 51,13 & $(7,11)$ & 49.75 & $(11.11)$ & 48.86 & $(6,70)$ \\
\hline investissement & 3,31 & 0.0423 & 57.30 & $(12,41)$ & 62.48 & $(3,01)$ & 61,91 & $(5.15)$ \\
\hline $\operatorname{ami}(\mathbf{e})$ & 0,11 & 0.8928 & 54,84 & $(13.28)$ & 56.61 & $(7,61)$ & 56,04 & $(8.82)$ \\
\hline \multicolumn{9}{|l|}{ exposition aux pairs } \\
\hline marginaux & 5.17 & 0.0080 & 69.72 & (7.62) & 75.86 & $(5,26)$ & 73,37 & $(7,70)$ \\
\hline \multicolumn{9}{|l|}{ Activités } \\
\hline argent de poche & 0.40 & 0.6692 & 50.29 & $(9.68)$ & 48.06 & $(8.24)$ & 49,31 & $(8,77)$ \\
\hline activités sociales & 0.70 & 0.4990 & 56,59 & $(13,89)$ & 59,98 & $(6.17)$ & 59.19 & $(7.90)$ \\
\hline activités passives & 1.70 & 0.1903 & 53.68 & $(10.66)$ & 49.85 & $(9,67)$ & 54.41 & $(8.96)$ \\
\hline activités de participation & 1,11 & 0.3343 & 46,95 & $(7,87)$ & 47,68 & $(9.43)$ & 50,98 & $(11,32)$ \\
\hline travail & 0,99 & 0.3758 & 48.01 & $(7,49)$ & 50.10 & $(9.37)$ & 52,82 & $(15.07)$ \\
\hline flânerie & 1.06 & 0.3502 & 51,69 & $(13,09)$ & 55,54 & $(13.79)$ & 50.06 & $(15,44)$ \\
\hline fréquentation d'arcades & 0.18 & 0,8330 & 73,28 & $(21,80)$ & 69,93 & $(17,13)$ & 72.03 & $(17,80)$ \\
\hline
\end{tabular}


Tableau 2 (suite)

\begin{tabular}{|c|c|c|c|c|c|c|c|c|}
\hline \multirow[b]{2}{*}{ Variables } & \multirow[b]{2}{*}{ F/X2 } & \multirow[b]{2}{*}{$\mathbf{p}$} & \multicolumn{2}{|c|}{$\begin{array}{c}\text { réseaux } \\
n=21\end{array}$} & \multicolumn{2}{|c|}{$\begin{array}{c}\text { quasi-bandes } \\
\mathbf{n}=\mathbf{3 1}\end{array}$} & \multicolumn{2}{|c|}{$\begin{array}{c}\text { bandes struct. } \\
\qquad \mathbf{n = 2 2} \\
\end{array}$} \\
\hline & & & moy. & é,-t. & moy. & é.-t. & moy. & é.t. \\
\hline \multicolumn{9}{|l|}{ SNormes } \\
\hline adhésion aux normes & 0.66 & 0,5224 & 43.14 & $(11.21)$ & 39,27 & $(15.25)$ & 38,75 & $(14,38)$ \\
\hline respect de l'autorité & 0.46 & 0.6303 & 35.96 & $(11.10)$ & 39.10 & $(12,48)$ & 36.94 & (12.38) \\
\hline neutralisation & 1.93 & 0.1525 & 48,82 & $(8.99)$ & 51,11 & (9.98) & 54.54 & $(9.76)$ \\
\hline \multicolumn{9}{|l|}{ Adaptation personnelle } \\
\hline mésadaptation sociale & 7,24 & 0.0015 & 56.54 & $(8.82)$ & 67.76 & $(11,58)$ & 68,88 & (12.01) \\
\hline autisme & 8.46 & 0.0006 & 54.30 & $(7,73)$ & 66.23 & (13.12) & 68,73 & $(11,58)$ \\
\hline aliennation & 4.67 & 0.0131 & 57.71 & $(9.51)$ & 64.08 & $(13.32)$ & 70.36 & (12.98) \\
\hline orientation aux valeurs & 8.58 & 0.0005 & 55.40 & $(7.72)$ & 66.28 & $(9,09)$ & 67,37 & $(12,47)$ \\
\hline repliement & 1,95 & 0.1513 & 45.31 & $(10,32)$ & 40,79 & $(5,30)$ & 42.60 & $(7,04)$ \\
\hline déni & 7,67 & 0,0011 & 46.95 & $(6,86)$ & 37.66 & $(7,78)$ & 38.68 & $(9,85)$ \\
\hline retrail & 5.27 & 0.0078 & 53.39 & $(8,79)$ & 62,18 & $(10,44)$ & 62,10 & $(9,15)$ \\
\hline agressivité manifeste & 6.50 & 0.0028 & 55.45 & (8.55) & 66.50 & $(10,61)$ & 63.49 & $(10.97)$ \\
\hline anxiété sociale & 2.74 & 0.0728 & 46.38 & $(7.04)$ & 53,44 & $(11,03)$ & 49,95 & $(10.76)$ \\
\hline psychotisme & 1,07 & 0.3503 & 77,14 & (10.69) & 82,08 & $(12.46)$ & 81.23 & $(10,43)$ \\
\hline extraversion & 0.68 & 0.5086 & 62.88 & $(6,35)$ & 61,36 & $(9.71)$ & 59.60 & $(7.58)$ \\
\hline névrotisme & 2,86 & 0,0651 & 50.43 & $(8,83)$ & 56.91 & $(10,33)$ & 52,44 & $(8,01)$ \\
\hline \multicolumn{9}{|l|}{ Troubles de comportement } \\
\hline troubles de componement & 3.59 & 0.0325 & 66.50 & (8.79) & 72,07 & $(11,16)$ & 74,94 & $(11.02)$ \\
\hline rébellion familiale & 3,56 & 0.0331 & 60.37 & $(11,84)$ & 64,80 & $(13,20)$ & 70.17 & $(10.39)$ \\
\hline inadaptation scolaire & 2.78 & 0.0689 & 53.87 & $(10.16)$ & 58.37 & $(10.38)$ & 60.76 & (8.29) \\
\hline promiscuité sexuelle & 0,18 & 0,8343 & 60.96 & $(7,04)$ & 60,91 & $(8.06)$ & 62,00 & $(4,95)$ \\
\hline drogues et alcool & 2.36 & 0.1017 & 64.65 & $(11.63)$ & 71.42 & $(14,25)$ & 73.56 & $(15.97)$ \\
\hline \multicolumn{9}{|l|}{ Délinquance } \\
\hline délinquance criminelle & 2,51 & 0,0888 & 64,46 & (17.76) & 73.23 & $(16,06)$ & 75,52 & $(18.30)$ \\
\hline agression & 5.19 & 0.0079 & 62.32 & $(15.47)$ & 71.43 & $(17.56)$ & 78.73 & $(16.65)$ \\
\hline vandalisme & 1.95 & 0.1495 & 59.07 & $(16.30)$ & 68.38 & $(12.29)$ & 71.39 & $(22.88)$ \\
\hline vol mineur & 0.47 & 0.6289 & 57.42 & $(15.07)$ & 60,45 & $(13.70)$ & 57,13 & (13,33) \\
\hline vol grave & 0.51 & 0.6051 & 65,21 & $(24,47)$ & 69,02 & $(24,63)$ & 72.81 & $(25,24)$ \\
\hline délinquance grave & 1,98 & 0.1457 & 65,69 & $(23.52)$ & 73,72 & $(23,14)$ & 79.76 & $(23,14)$ \\
\hline
\end{tabular}

également une expérience semblable à l'école. Bien que l'école ne représente pas une source de malaise, les liens tissés avec les enseignants sont fragiles et l'importance de la scolarisation n'est pas pleinement reconnue. De plus, peu importe la structure des bandes auxquelles elles adhèrent, les filles ne se conforment pas aux attentes des enseignants puisqu'elles sont la cible de nombreuses sanctions.

Les relations avec les pairs distinguent davantage les groupes. Les filles qui adhèrent aux quasi-bandes sont celles qui consacrent le plus de temps à leurs pairs $(F=3,31 ; d l=2 ; p<0,05)$. Ces pairs n'incitent toutefois pas à la conformité sociale, car plusieurs d'entre eux ont déjà eu des contacts avec la police $(F=5,17 ; d l=2 ; p<0,01)$. Quant aux activités que pratiquent les membres de chaque niveau de structure, elles se comparent. Toutes allouent un temps raisonnable aux activités sociales et culturelles, de même qu'au 
travail rémunéré et à la flânerie. Les filles ne sont toutefois pas à l'abri des influences marginales, car elles fréquentent régulièrement les arcades. Enfin, la réceptivité aux contraintes sociales est faible pour les membres des trois niveaux de structure. Les filles membres des réseaux tout comme celles des quasi-bandes et des bandes structurées adhèrent peu au système normatif de la société. Les trois groupes d'adolescentes respectent également peu les individus en position d'autorité.

Tout compte fait, les difficultés d'adaptation sociale augmentent peu au fur et à mesure que l'organisation de la bande s'accentue. Dans l'ensemble, le profil des filles se compare peu importe la structure des bandes auxquelles elles participent. En effet, la structure de la bande n'affecte pas la qualité des liens qui sont noués avec les différentes institutions. De plus les contraintes auxquelles les adolescentes sont confrontées ainsi que les barrières morales qu'elles s'imposent demeurent imperméables à l'influence de la structure de la bande. L'exposition aux influences déviantes s'accrô̂t toutefois lorsque la structure de la bande se définit. Des résultats similaires s'observent chez les garçons (Le Blanc et Lanctôt, 1996).

\section{ADAPTATION PERSONNELLE}

Contrairement à leur adaptation sociale, la personnalité des filles se distingue selon le degré d'organisation des bandes auxquelles elles adhèrent. Les membres des quasi-bandes et les membres des bandes structurées ont une personnalité lourdement marquée comparativement à celle des membres des bandes les moins organisées.

Les filles des deux niveaux de structure les plus élevés ont de faibles habiletés à rencontrer les exigences sociales (mésadaptation sociale : $F=$ 7,$24 ; d l=2 ; p<0,01$ ). Ces adolescentes donnent la priorité à l'opportunisme et à l'opposition, ce qui ternit leurs relations interpersonnelles. L'orientation délinquante des membres des quasi-bandes et des bandes structurées repose de plus sur une façade fabulée. L'autisme $(F=8,46 ; d l=2 ; p<0,001)$ amène ces filles à déformer la réalité selon leurs propres besoins et désirs. Ces filles ajoutent ainsi de la couleur à leur réalité afin de se croire en contrôle de leurs moyens. De surcroît, la personnalité de ces membres des bandes est envahie par une dynamique de suspicion. Les résultats élevés à l'aliénation $(\mathrm{F}=4,67 ; d l=2 ; p<0,05)$ indiquent qu'elles se montrent méfiantes à l'endroit des individus en autorité. Leur rapprochement avec autrui devient une fois de plus difficile. L'orientation grégaire est également plus marquée chez les membres des bandes les plus structurées. L'orientation aux valeurs délinquantes $(F=8,58 ; d l=2 ; p<0,001)$ amène ces filles à se forger une image de dures. 
Les expériences interpersonnelles sont donc nettement appauvries chez les filles membres des quasi-bandes et des bandes structurées. Le climat intérieur de ces filles est également malsain. Leur résultat faible au déni ( $F=$ 7,$67 ; d l=2 ; p=0,001$ ) trahit une difficulté à pondérer les conflits, ce qui les porte à dramatiser les événements. Le retrait $(F=5,27 ; d l=2 ; p<0,05)$ reflète quant à lui une tendance à s'isoler des autres à cause d'un manque de satisfaction à l'égard de soi-même et à l'égard d'autrui. Contrairement aux membres des réseaux, les filles qui participent à des bandes davantage structurées préfèrent fuir les rapports interpersonnels. Enfin, l'agressivité manifeste est marquée chez les membres des quasi-bandes $(F=6,50 ; d l=2 ; p<$ $0,05)$. Ces filles sont vulnérables aux comportements explosifs. Elles se sentent impuissantes et elles sont prêtes à réagir fortement à la pression intérieure qu'elles éprouvent.

En somme, l'adaptation personnelle des filles membres des quasi-bandes et des bandes structurées accuse de lourds déficits alors que celle des membres des réseaux se veut davantage dass la norme. Cette dynamique ne se retrouve pas chez les garçons. Leur personnalité accuse des déficits du même ordre d'un niveau de structure à l'autre (Le Blanc et Lanctôt, 1996). Chez les filles, faut-il avoir des difficultés personnelles sérieuses pour adhérer à une bande avec un certain degré de structure ?

\section{ACTIVITÉS MARGINALES}

Même si la personnalité des filles des bandes les plus structurées trahit un réel potentiel de passage à l'acte, les activités marginales de ces dernières se démarquent très peu de celles des membres des réseaux. Quelques différences sont toutefois perceptibles, notamment au niveau des troubles de comportement. Les membres des bandes structurées affichent la moyenne la plus élevée à l'indice général de la déviance $(F=3,59 ; d l=2 ; p<0,05)$ ainsi qu'à la rébellion familiale $(F=3,56 ; d l=2 ; p<0,05)$. Toutefois, aucune différence significative ne s'observe pour l'inadaptation scolaire, la promiscuité sexuelle et la consommation de psychotropes.

Du côté de la đélinquance, seule l'agression physique distingue les membres des trois groupes. Les filles qui participent aux bandes structurées ont commis divers comportements d'agression au cours de la dernière année ( $F$ $=5,19 ; d l=2 ; p<0,01$ ). Une analyse détaillée montre que ces actes d'agression se traduisent principalement par la participation aux batailles entre groupes de jeunes $(F=3,93 ; d l=2 ; p<0,05)$, par l'utilisation d'une arme lors d'une bataille $(F=5,12 ; d l=2 ; p<0,01)$ et par le port d'une arme $(F=$ $3,84 ; d l=2 ; p<0,05)$. Ces comportements sont d'ailleurs réputés fréquents chez les filles membres des bandes. De nombreux auteurs rapportent en effet que la dynamique de la bande laisse peu d'initiatives délinquantes aux filles, 
mis à part la participation aux batailles et le transport des armes (Haskins, 1974 ; Bowker, 1980, Campbell, 1984b ; Fishman, 1988 ; Joe et ChesneyLind, 1995). Les filles occupent ainsi des rôles d'auxiliaires et elles soutiennent les garçons lors des confrontations avec les bandes rivales. Les données du tableau 2 confirment donc que la dynamique des bandes structurées incite les membres féminins à participer aux batailles et à transporter des armes. Cependant, les données démontrent également que la délinquance des filles qui participent aux bandes ne se limite pas à ces comportements. En effet, les actes de vandalisme, les vols mineurs, les vols graves ainsi que la délinquance grave ne sont pas rares chez les adolescentes, peu importe le degré de cohésion qui s'installe au sein de leur bande.

La structure de la bande exerce donc une mince influence sur les activités marginales des adolescentes, puisque seuls les actes d'agression sont perméables à la dynamique des bandes. Cette conclusion est renforcée par les résultats de l'analyse discriminante. Au total, le modèle retient neuf prédicteurs qui expliquent $22 \%$ de la variance. Seules deux variables du profil comportemental sont capables de discriminer les membres des bandes structurées des membres des bandes les moins organisées, et leur importance relative est faible. Il s'agit de la rébellion familiale et de l'agression physique. Les filles qui adhèrent aux bandes structurées se démarquent davantage par les déficits de leur personnalité, puisque la variable du modèle multivarié qui discrimine le plus fortement les groupes est l'autisme. Ainsi, les filles qui adhèrent aux bandes structurées se caractérisent principalement par le recours à une façade d'efficacité afin de se donner du pouvoir. Cette propension à structurer artificiellement la réalité est également observée par Arpin et coll. (1994) :

Toute la soumission, l'exploitation servile et la dévalorisation sont massivement projetées à l'extérieur et attribuées aux autres filles de la bande. L'image de soi est pauvre et construite défensivement autour d'une façade où l'on camoufle qui on est vraiment et où l'on se donne l'illusion d'être grande. (Arpin et coll., 1994, p.10).

L'analyse discriminante reconnaît donc l'importance des déficits de la personnalité des membres des bandes structurées. La structure de la bande se prête ainsi à un mécanisme de sélection, puisque la dysfonctionnalité personnelle des filles augmente au fur et à mesure que la cohésion de la bande s'accentue. Le modèle multivarié suggère cependant que ce potentiel s'actualise très peu dans le contexte de bande. D'une part, la dysfonctionnalité des filles des bandes structurées se décharge surtout contre la famille, par la rébellion familiale. D'autre part, l'organisation de la bande semble avoir pour effet de confiner les filles à des rôles d'auxiliaires plutôt que de leur permettre d'actualiser leur potentiel antisocial par la manifestation d'actes graves. 
En effet, les filles qui adhèrent aux bandes structurées se démarquent des autres membres, non pas au niveau de la délinquance grave, mais au niveau du port des armes et de la participation aux batailles. Chez les garçons, les résultats sont complètement différents. D'abord, la personnalité de ces derniers varie peu selon la structure de la bande. L'adhésion des garçons aux bandes structurées semble plutôt découler des opportunités de leur milieu. Ensuite, la cohésion de la bande a davantage d'influence sur leurs activités délinquantes, car l'agression est un comportement qui discrimine fortement les garçons membres des bandes structurées des garçons des bandes moins organisées (Lanctôt et Le Blanc, 1996b).

\section{CONCLUSION}

L'analyse du profil des adolescentes qui adhèrent aux bandes marginales suggère une explication différente de celle qui se retrouve généralement dans la littérature. L'utilisation d'un sondage démontre d'abord que plus de deux adolescentes en difficulté sur trois ont déjà adhéré à une bande. Nos résultats jndiquent de plus que les filles qui participent aux bandes ont de réelles difficultés d'adaptation sociale et personnelle. Ces adolescentes ne sont pas à l'abri des influences déviantes, puisqu'elles fréquentent des pairs et des lieux marginaux. Cette exposition à la marginalité est facilitée par une surveillance parentale relâchée. Les membres des bandes s'imposent également peu de contraintes internes. Leur personnalité rend quant à elle difficiles les rapprochements avec autrui. Les adolescentes en difficulté ne s'associent pas aux bandes que parce qu'elles ont besoin d'être comprises et aidées ou parce qu'elles ont une image pauvre d'elles-mêmes. Comme les garçons qui font partie des bandes, elles ont besoin de contrer, de provoquer autrui et elles font preuve de peu de savoir-faire social. Le profil comportemental indique enfin que les filles membres des bandes ne se caractérisent pas seulement par leurs troubles de comportement, la délinquance occupant également une place importante dans le répertoire de leurs activités. L'agression physique est d'ailleurs le prédicteur numéro un de l'adhésion des filles aux bandes marginales. Ce profil des membres féminins se rapproche grandement des caractéristiques observées chez les garçons membres des bandes (Lanctôt et Le Blanc, 1996b).

L'analyse rapporte toutefois que l'influence des bandes n'est pas la même pour les garçons et pour les filles. La personnalité des filles se détériore au fur et à mesure que l'organisation de la bande se définit, alors que chez les garçons, cet enfoncement de plus en plus marqué dans la dysfonctionnalité ne s'observe pas (Le Blanc et Lanctôt, 1996). Le modèle multivarié confirme ce processus de sélection des filles qui adhèrent à des bandes structurées. L'autisme s'avère le prédicteur qui discrimine le plus 
fortement les membres de chaque niveau de structure. Les filles qui participent aux bandes structurées se caractérisent donc principalement par leur tendance à s'inventer un pouvoir d'efficacité. La présence de fortes perturbations chez ces adolescentes ne se traduit toutefois pas par une délinquance davantage diversifiée que celle des membres des réseaux. Seules la rébellion familiale et l'agression physique se retrouvent avec plus de variété dans le répertoire des membres des bandes structurées. Cette observation laisse supposer que la dynamique des bandes structurées atténue le potentiel antisocial des filles. En effet, l'organisation de la bande ne permet pas aux filles de décharger leurs tensions et leurs frustrations, mais favorise plutôt l'occupation de rôles secondaires. Le peu d'initiatives délinquantes que la dynamique de la bande octroie aux filles a d'ailleurs été maintes fois signalé (Haskins, 1974 ; Bowker, 1980, Campbell, 1984b ; Fishman, 1988 ; Joe et Chesney-Lind, 1995).

D'autre part. nos résultats apportent des informations qui méritent de ne pas être négligées. La faible influence dont fait preuve la structure des bandes sur les divers comportements des membres féminins ne signifie pas que les troubles de comportement et la délinquance occupent peu de place dans le mode de vie de ces adolescentes. Les résultats, qui dépassent largement le corridor de la normalité, indiquent plutôt que les membres des bandes commettent une variété d'actes marginaux, des plus bénins aux plus graves. Ces résultats peuvent porter à confusion. Les filles qui s'impliquent dans les bandes commettent une variété d'actes marginaux alors que la dynamique de la bande semble limiter leurs activités déviantes et délinquantes. La dysfonctionnalité des filles se manifesterait-elle surtout hors du contexte des bandes ? Cette question mériterait d'être approfondie. Il faudrait alors distinguer les agirs qui se commettent dans le contexte de la bande de ceux qui se manifestent indépendamment de celui-ci. D'ici là, il faut retenir que les adolescentes judiciarisées et membres des bandes, et surtout celles qui s'affilient à des bandes structurées, ont de réelles difficultés à fonctionner en harmonie avec la société. En ceỉa, elles ne sont pas différentes des pupilles mâles du tribunal.

\section{BIBLIOGRAPHIE}

ACKLEY, E. G. ET FLIEGEL, B. R. (1960), « A Social Work Approach to Street-Corner Gir]s », Social Work, vol. 5, n 4, pp. 27-36.

ARPIN, R. DUBOIS, R. DULUDE, D. ET BISAILLON, C. (1994), «Étude exploratoire du phénomène d'appartenance à la bande chez l'adolescente dite "mésadaptée socioaffective" ", Revue canadienne de psycho-éducation, vol. 23, n 1, pp. 1-15.

BJERREGAARD, B. ET SMITH, C. (1993), «Gender Differences in Gang Participation and Delinquency ", Journal of Quantitative Criminology, vol. $9 \mathrm{n}^{\circ} 4$, pp. 329-355 
BROTHERTON, D. C. (1996), « "Smartness," “Toughness,” and “Autonomy” : Drug Use in the Context of Gang Fernale Delinquency », Journal of Drug Issues, vol. 26, $\mathrm{n}^{\circ} \mathrm{l}$, pp. 261-277.

BOWKER, L. H. GROSS, H. S. ET KLEIN, M. W. (1980), « Female Participation in Delinquent Gang Activities », Adolescence, vol. $x \vee, n^{\circ} 59$, pp. 509-519.

BOWKER, L. E. ET KLEIN, M. (1983), « The Etioloy of Female Juvenile Delinquency and Gang Membership : A Test of Psychological and Social Structural Explanations », Adolescence, vol. $18 \mathrm{n}^{\circ} 72$, pp. 739-751.

BROWN, W. K. (1977), « Black Female Gangs in Philadelphia », International Journal of Offender Therapy and Comparative Criminology, vol. 21, $\mathrm{n}^{\circ} 3$, pp. 221-228.

CAMPBELL, A. (1984a), « Girl's Talk : The Social Representation of Agression by Female Gang Members », Criminal Justice and Behavior, vol. 11, n 2, pp. 139-156.

CAMPBELL, A. (1984b), The Girl's in the Gangs, Blackwell, Oxford.

CAMPBELL, A. (1990), « On the Invisibility of the Female Delinquent Peer Group », Women \& Criminal Justice, vol. 2, $\mathrm{n}^{\circ} 1, \mathrm{pp} .41-62$.

CLOWARD, R. A. ET OHLIN, L. E. (1960), Delinquency and Opportunity : A Theory of Delinquent Gangs. Gleancoe, The Free Press.

COHEN, A. K. (1955), Delinquent Boys: The Culture of the Gang, Glencoe, The Free Press.

COLLINS, H. C. (1979), Street Gangs, Profiles for Police, New York : Office of Community Affairs Youth Aid Division, City of New York, Police Department.

CRAWFORD, P. I., MALAMUD. D. I. ET DUMPSON, J. R. (1950), Working with Teenage Gangs, New York, Welfare Council.

FAGAN, J. (1990), « Social Processes of Delinquency and Drug Use Among Urban Gangs », in C. R. HUFF (éd.) Gangs in America, Newbury Park, Sage, pp. 183-222.

FISHMAN, L. T. (1988), The Vice Queens : An Ethnographic Study of Black Female Gang Behavior, communication présentée lors des congrès annuel de l'American Society of criminology.

HARRIS, M. G. (1988), Cholas, Latino Girls and Gangs, New York, AMS Press.

HASKINS. J. (1974), Sireet Gangs, Yesterday and Today, New York, Hastings House.

JOE, K. A. et CHESNEY-LIND, M. (1995), « Just Every Mother's Angel. An Analysis of Gender and Ethnic Variations in Youth Gang Membership », Gender and society, vol. 9, $\mathrm{n}^{\circ} 4$, pp. $408-431$.

LANCTÔT, N. et LE BLANC, M. (1996a), « Filles et garçons membres de bandes marginales ", Les Adolescents en difficulté des années 1990. Rapport $n^{\circ} 6$. Groupe de recherche sur les adolescents en difficulté. École de Psycho-Éducation, Université de Montréal.

LANCTÔT, N et LE BLANC, M. (1996b), « La participation des garçons aux bandes marginales : Un phénomène de sélection et d'opportunités ", Revue canadienne de criminologie.

LE BLANC, M. et LANCTÔT, N. (1996), «Adolescent Gang Members Social and Psychological Characteristics, Gang Participation a Selection or an Activation Process? ", Journal of Gang Research.

LE BLANC, M. McDUFF, P. et FRÉCHETTE, M. (1994), MASPAQ, Manuel sur des mesures de l'adaptation sociale et personnelle pour les adolescents québécois. Groupe de recherche sur l'inadaptation psycho-sociale à l'enfance, Université de Montréal. 
MILLER, W. B. (1958), « Lower Class Culture as a Generating Milieu of Gang Delinquency », Journal of Social Issues, vol. 14, $\mathrm{n}^{\circ}$ 3, pp. 5-19.

MILLER, W. B. (1973), «The Molls », Society, vol. 11, n 1, pp. 32-35.

MILLER, W. B. (1975), Violence By Youth Gangs And Youth Groups As A Crime Problem In Major American Cities, Mass, Center for Criminal Justice Harvard Law School Cambridge.

SARNECKI, J. (1986), Delinquant Network, Suède, The National Council for Crime Prevention.

SHORT, J. F. (1968), Gang Delinquency and Delinquent Subcultures, New York, Harper \& Row.

TAYLOR, C. S. (1990), Dangerous Society, East Lansing, Michigan, Michigan State University Press.

THRASHER, F. (1927), The Gangs, Chicago, University of Chicago Press. 